\title{
Acoustic Beam Scanning Using Annular Transducer Array Introducing Decentering Operation*
}

\author{
Hiroyuki MASUYAMA** and Koichi MIZUTANI ${ }^{* * *}$
}

\begin{abstract}
A method for scanning a radiated acoustic beam is described, by introducing a concise decentering operation in an annular transducer array sound source. For generating a narrow acoustic beam, we have proposed the simple method of using a concentric annular array in which the width of each element is weighed by the $J_{0}$ function. Additionally, by applying a clearance that exists between each neighboring element of the array to decenter the array elements, we have shown that the direction of the radiated acoustic beam is made to be variable. Even when this decentering procedure is optimally, the shifting of the propagation path of the beam still occurs, and it is possible to scan the beam precisely. Using numerical calculations, we show the scanned aspect of the narrow beam generated by this method. The validity and applicability of this method are then examined.
\end{abstract}

Key Words: Acoustic, Sound Field Control, Measurement, Annular Transducer Array, Nondiffracting Beam, Scanning, Decentering Operation

\section{Introduction}

Acoustic waves are widely used in measuring equipment and imaging devices, because of features such as noninvasiveness and ease of handling. To improve the performance of these equipment and devices and to obtain an output with a high transverse resolution, it is desired that acoustic beams with a narrow width are used and that the beam which keeps a sharp profile is propagated. Since the width of acoustic beams radiated in propagating space generally extends owing to diffraction, it is difficult for a narrow beam to propagate over a long distance.

However, the optical formation method for a nondiffracting beam was introduced by Durnin ${ }^{(1),(2)}$ in 1987 , and the acoustic method for forming such a nondiffracting beam in which the radiation direction is narrowed down has been widely studied ${ }^{(3)-(8)}$.

As one of them, a method that uses an equiamplitudedriven annular transducer array has been proposed ${ }^{(9),(10)}$. Since this method has a much simpler structure than the

* Received 2nd February, 2006 (No. 05-4296)

** Electronic Mechanical Engineering Department, Toba National College of Maritime Technology, 1-1 Ikegami-cho, Toba 517-8501, Japan.

E-mail: masuyama@toba-cmt.ac.jp

*** Graduate School of Systems and Information Engineering, University of Tsukuba, Tsukuba Science City 305-8573, Japan. E-mail: mizutani@esys.tsukuba.ac.jp conventional methods, it seems to be useful as one of the methods for forming the above-described nondiffracting beam approximately. Additionally, an array of this type has the feature that there exists a clearance between each neighboring element. By utilizing these clearances to decenter array elements, the sound source using this method makes the direction of the radiated ultrasonic beam variable ${ }^{(11)-(13)}$. Additionally, it is shown that there is a relationship between the actually decentered array element and the sound axial distance over which the direction of the formed beam changes ${ }^{(14)}$. Furthermore, it is shown that the ratio of the decentering amount of each array element corresponding to a certain distance from the sound source acts on the radiation direction of the beam there, and that the whole profile of the formed beam is changed by combining these effects ${ }^{(15)-(17)}$.

In this paper, we present a method for realizing a sound source in which the radiating position of the beam is scanned, introducing the most simplified decentering operation. In other words, only one ring element that is next to the center disk element is used for the decentering operation, and the aspect of the radiation beam in this case is examined using numerical calculations.

In the following section, the design method for a concentric annular transducer array that radiates an acoustic beam with a sharp profile is shown. Successively, the decentering procedure for the sound source element for scanning the position of the radiated beam is described. After 
that, the results of numerical calculations of the radiated sound field are shown. From the results, it is shown that the scanned aspect of the acoustic beam is generated by the simple technique, and that the center of the radiated beam moves in proportion to the ratio of the decentering amount of the singular decentered element. Conclusions are given in the last section.

\section{Design Method for Sound Source with Scanning Function}

\subsection{Concentric annular transducer array for forming narrow beam}

Let us consider a coordinate system on which a sound source consisting of an annular transducer array is allocated, as shown in Fig. 1. The sound source is allocated on a plane perpendicular to the $z$-axis, where $r$ is the radial distance from the $z$-axis. To begin with, the formulation method for a concentric annular transducer array, shown in Fig. 1, is explained using Fig. 2.

Figure 2 (a) shows a circular transducer driven with a normal velocity distribution represented by the zerothorder Bessel function of the first kind, $J_{0}$. The beam radiated from such a transducer is known as the "Bessel beam". This beam has a narrow intensity profile and propagates for a certain distance without diffractive spreading. Here, we consider the approximation of this ideal sound source consisting of a concentric annular transducer array, in which each element has a width corresponding to the normal velocity distribution shown in Fig. 2 (a).

The location and width of each element of the sound source are decided by the zeroth-order Bessel function of the first kind, $J_{0}(x)$, which is shown in Fig. 2 (b). The center disk element is located at $r=0$, which yields the main extreme value of the $J_{0}$ function, and $r_{n}$, the center radius of the $n$th element from the innermost disk element, is arranged in $r$, which yields the $n$th extreme value of the scaled $J_{0}$ function, $J_{0}(\alpha r)$, as shown in Fig. 2 (c). In the initial stage, the width of each element is relatively determined by the area $S_{n}$ in Fig. 2 (b) enclosed by the extreme lobe of the $J_{0}$ function and the $x$-axis.

When the center disk element and the second ring element are close together, as shown in Fig. 2 (d), the width of each element becomes the largest possible value. When the radius of the center disk element in such a case is $D$, the actual radius the center disk element $d$, as shown in Fig. 2 (e), is given by $d=K D$, where $K$ is the coefficient that determines the absolute width of each array element, which is decided only relatively by the $J_{0}$ function. Finally, a concentric annular array sound source, shown in Fig. 2 (f), is formulated. The beam which has a sharp profile and propagates without diffractive spreading is radiated by driving a source formed in this way with an antiphase from its neighboring elements and with an equiamplitude.

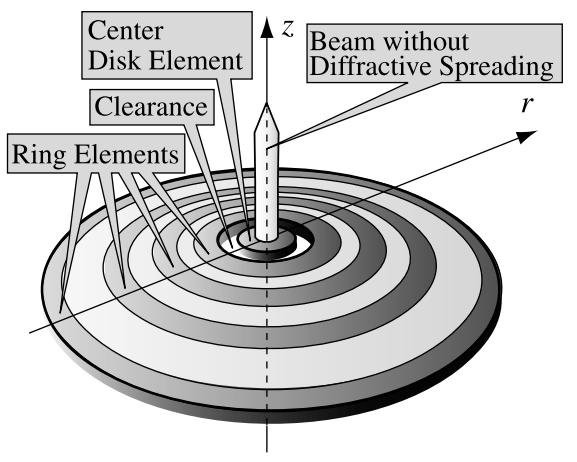

Fig. 1 Geometry of coordinate system, sound source consisting of concentric annular transducer array and radiated beam without diffractive spreading

(a)
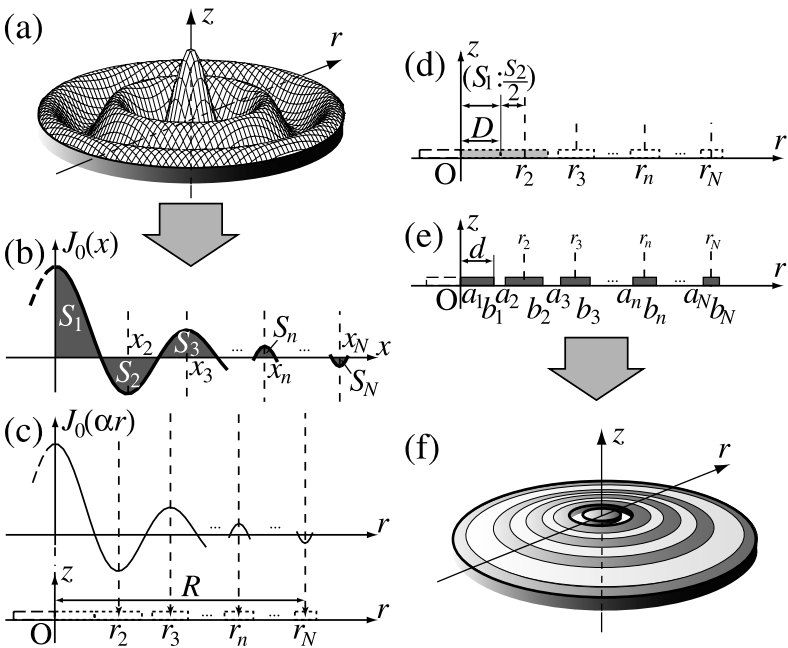

Fig. 2 Design procedure for concentric annular array for generating nondiffracting beam. (a) Circular transducer driven with normal velocity distribution represented by zeroth-order Bessel function of the first kind, $J_{0}$, (b) zeroth-order Bessel function of the first kind, $J_{0}(x)$, (c) allocation of center radius of each element, $r_{n}$, (d) maximum radius of center element, $D$, (e) actual radius of center element, $d$, and allocations of inner radius, $a_{n}$, and outer radius, $b_{n}$, of each element and (f) concentric annular transducer array in which each element has width corresponding to $J_{0}$ function

The parameters for constituting this annular array sound source, $r_{n}, \alpha, D, d, a_{n}, b_{n}$, are given by the following equations.

$$
\begin{aligned}
& r_{n}=R \frac{x_{n}}{x_{N}} \quad(n>2), \\
& \alpha=\frac{x_{N}}{r_{N}}, \\
& D=r_{2} \frac{S_{1}}{S_{1}+\frac{S_{2}}{2}}, \\
& d=K D \quad(0<K \leq 1), \\
& a_{n}= \begin{cases}0 & (n=1), \\
r_{n}-d \frac{S_{n}}{2 S_{1}} & \text { (otherwise), }\end{cases}
\end{aligned}
$$


(a)

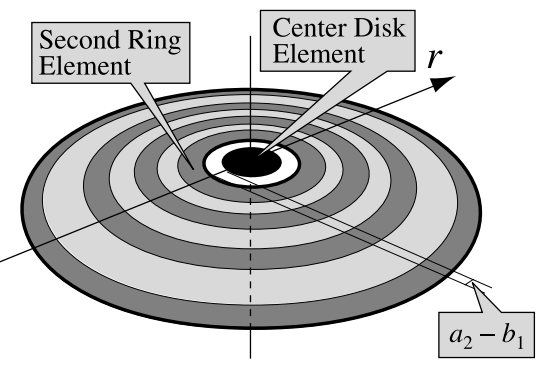

(b)

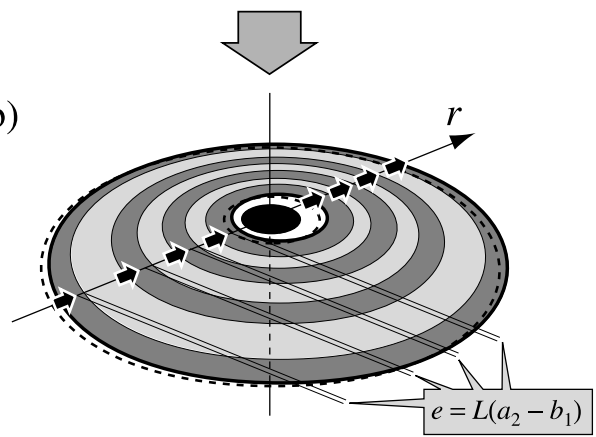

Fig. 3 Formation procedure for decentered annular transducer array. (a) Initial condition. (b) The second ring element and elements that are located further outside than it are moved at $e$

$$
b_{n}= \begin{cases}d & (n=1), \\ r_{n}+d \frac{S_{n}}{2 S_{1}} & \text { (otherwise) }\end{cases}
$$

Here, $R=r_{N}$ is the center radius of the outermost $N$ th element of the sound source, and $\alpha$ is the scale factor of the $J_{0}$ function.

\subsection{Decentering procedure for scanning radiating position of beam}

The decentering procedure for a ring array element for moving the center of the radiated beam is illustrated in Fig. 3. The clearances in the array are expressed by the inner radii $a_{n}$ and outer radii $b_{n}$ of array elements, and the width of the clearance between the center disk element and the second ring element becomes $a_{2}-b_{1}$. This width is the maximum possible value for decentering the second ring element, and the actual decentering amount $e$ corresponding to the second ring element is given by the following equation:

$$
e=L\left(a_{2}-b_{1}\right),
$$

where $L$ is a coefficient that is determined arbitrarily from the range of $0 \leq L \leq 1$, and will be termed the "decentering ratio" of the array element. As shown in Fig. 3(b), the second ring element is decentered at $e$ and, simultaneously, array elements that are located further outside than the second ring element are moved. As a result, a decentered annular transducer array radiating a positionscanned beam, shown in Fig. 4 , is constructed.

\section{Numerical Calculations}

The calculation results of the radiated pressure magnitude distributions, when the decentered annular trans-

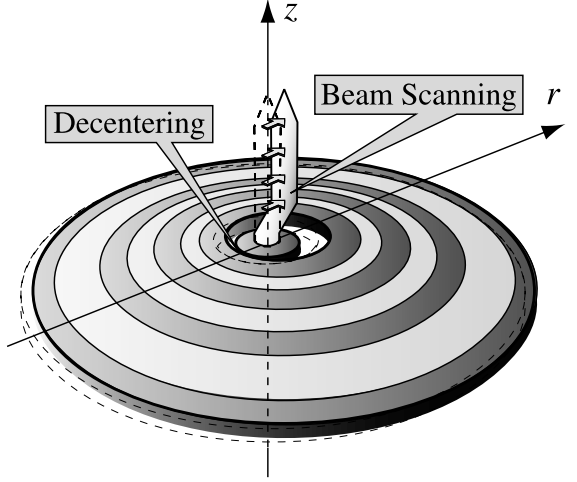

Fig. 4 Sound source consisting of decentered annular transducer array and radiated beam with direction scanning function

ducer arrays shown in the previous section are driven by a single-frequency continuous wave, are shown in Figs. 5 and 6 . Here, the wavelength of the driving signal is expressed as $\lambda$, and the distance using the calculation is normalized by $\lambda$. Figure 5 shows the cross-sectional amplitude distributions in the plane corresponding to the direction of decentering. Three-dimensional plots are shown in the upper part of subfigures, and top views are shown in the lower part of subfigures, as intensity distributions. Figure 6 shows the entire shape of the radiated beams in the three-dimensional space, as intensity distributions. Each array is driven by the single-frequency continuous wave with an antiphase from its neighboring elements. The number of array elements is 10 , the center radius of the outermost element $R$ is $10 \lambda$ and the $K$ of Eq. (4) is 0.6. Table 1 shows the values of the inner radius $a_{n}$, center radius $r_{n}$ and outer radius $b_{n}$ of each element of the array used in the calculation. For three-dimensional plots in Fig. 5, the intervals of the calculation point are $0.2 \lambda$ in the $r$-axial direction and $0.4 \lambda$ in the $z$-axial direction, and, for intensity distributions in Fig. 5, the intervals of the calculation point are $0.05 \lambda$ in the $r$-axial direction and $0.1 \lambda$ in the $z$-axial direction. For Fig. 6, the calculation intervals are $0.4 \lambda$ in both the $r$ - and $z$-axial directions. In Figs. 5 and 6 , the results obtained using six kinds of arrays of which the decentering ratio $L$ expressed by Eq. (7) differs are presented, and $L$ is set in each subfigure as (a) $L=0.0$ (not decentered), (b) $L=0.2$, (c) $L=0.4$, (d) $L=0.6$, (e) $L=0.8$ and (f) $L=1.0$. In three-dimensional plots in Fig. 5, the plot in each subfigure is normalized by the maximum value of the result shown in Fig. 5 (a). In intensity distribution in Figs. 5 and 6, the plot in each subfigure is normalized by the maximum value of each result. In addition, the directions of the decentering are identical in all cases. From each result in Figs. 5 and 6, it is shown that the radiated beams maintain a narrow width in all cases, and that the significant change in the beam amplitudes due to the decentering operation does not appear. Additionally, 
(a)
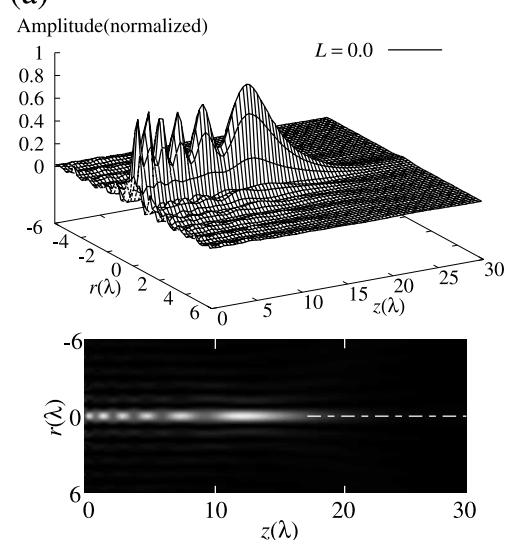

(d)
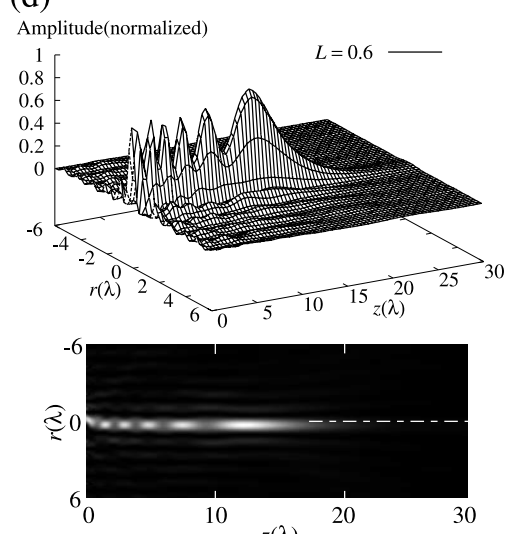

(b)
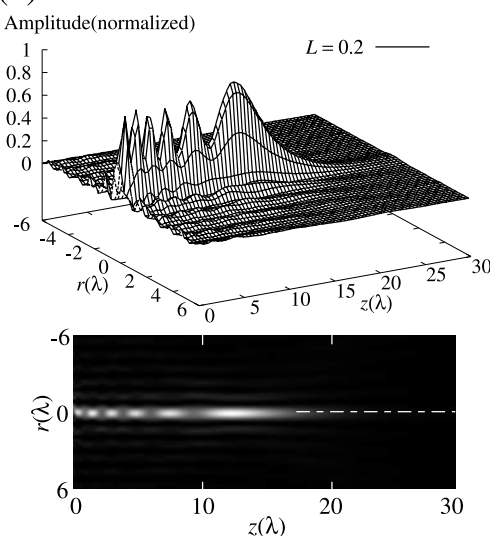

(e)
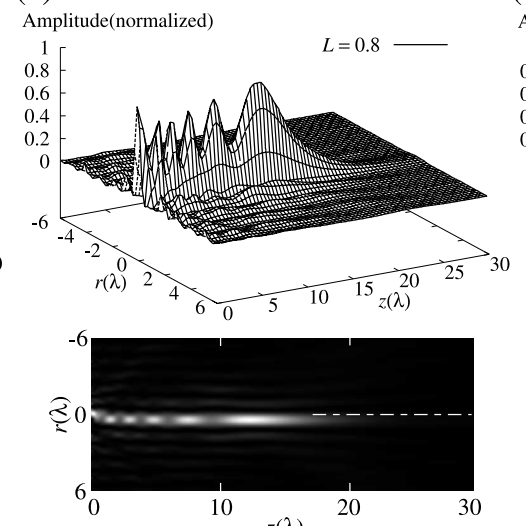

(c)
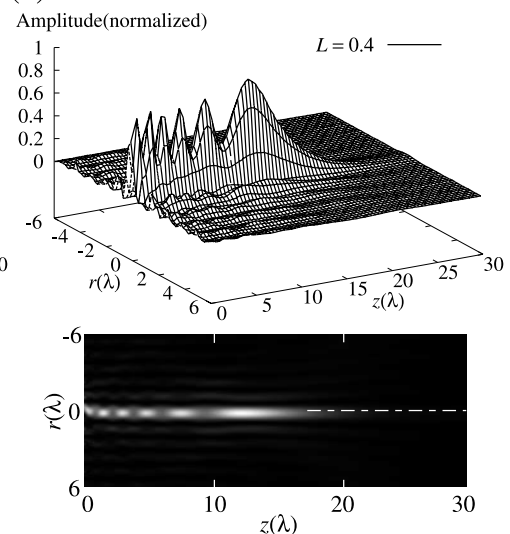

(f)
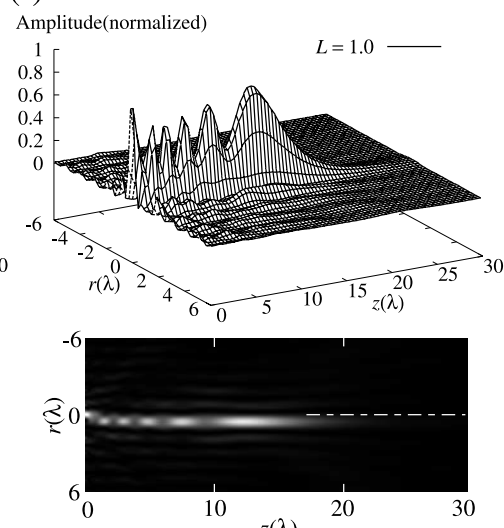

Fig. 5 Calculation results of magnitude of pressure radiated from decentered annular arrays, shown as three-dimensional plots (upper) and as intensity distributions of top view (lower) of cross-sectional amplitude distribution; 10 elements, $R=10 \lambda, K=0.6$; (a) $L=0.0$ (not decentered), (b) $L=0.2$, (c) $L=0.4$, (d) $L=0.6$, (e) $L=0.8$ and (f) $L=1.0$

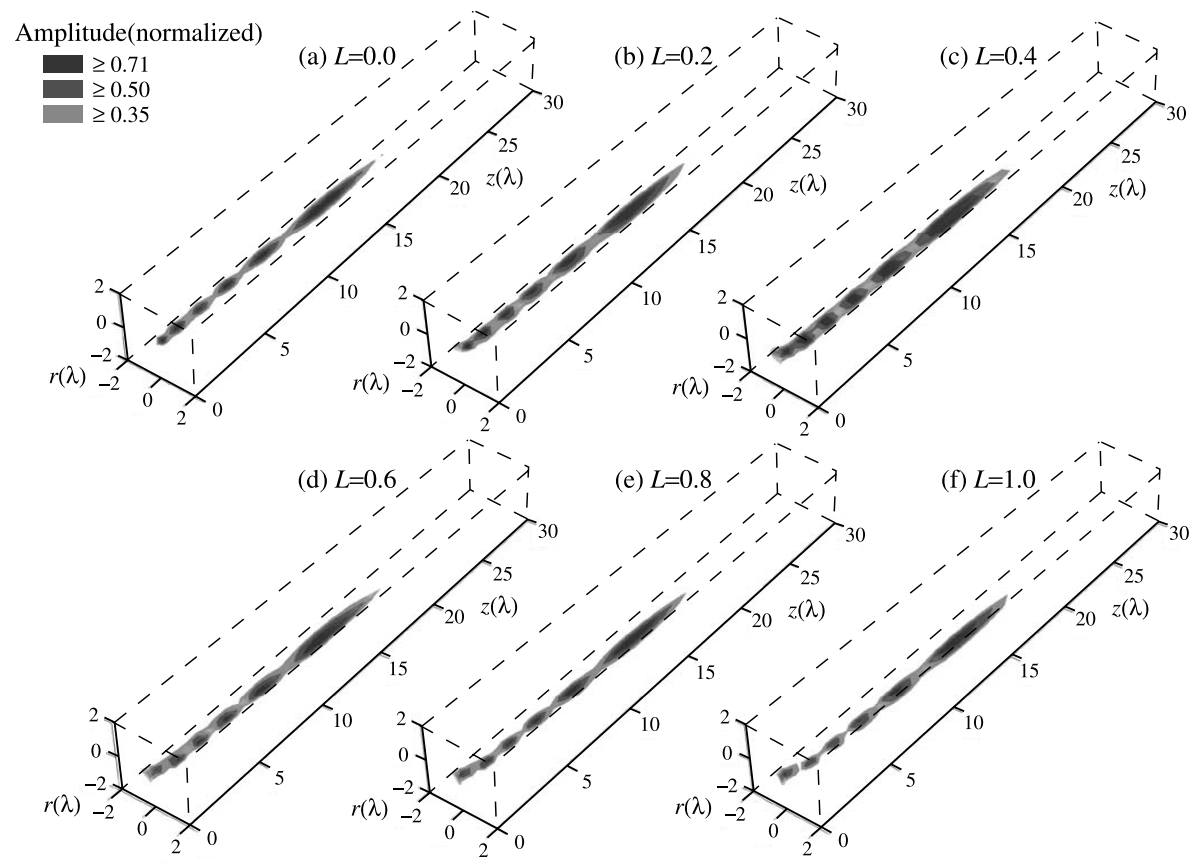

Fig. 6 Calculation results of magnitude of pressure radiated from decentered annular arrays, shown as intensity distributions in three-dimensional space; 10 elements, $R=10 \lambda$, $K=0.6$; (a) $L=0.0$ (not decentered), (b) $L=0.2$, (c) $L=0.4$, (d) $L=0.6$, (e) $L=0.8$ and (f) $L=1.0$ 
Table 1 Values of inner radii $a_{n}$, center radii $r_{n}$, and outer radii $b_{n}$ of each element of array used in calculation

\begin{tabular}{cccccc}
\hline$n$ & 1 (center disk) & 2 & 3 & 4 & 5 \\
\hline$a_{n}(\lambda)$ & 0.00000000 & 1.14962904 & 2.28851713 & 3.39690287 & 4.49464540 \\
$r_{n}(\lambda)$ & - & 1.31914703 & 2.41527466 & 3.50245122 & 4.58697688 \\
$b_{n}(\lambda)$ & 0.62197023 & 1.48866502 & 2.54203219 & 3.60799957 & 4.67930836 \\
\hline$n$ & 6 & 7 & 8 & 9 & 10 \\
\hline$a_{n}(\lambda)$ & 5.58727122 & 6.67704492 & 7.76494719 & 8.85161664 & 9.93739870 \\
$r_{n}(\lambda)$ & 5.67036645 & 6.75320518 & 7.83566521 & 8.91791867 & 10.00000000 \\
$b_{n}(\lambda)$ & 5.75346167 & 6.82936543 & 7.90638322 & 8.98422070 & 10.06260130 \\
\hline
\end{tabular}

Table 2 Slippage amount from $r=0$ of each radiated beam, $\Delta r$ (The average of eleven calculation results around $z=$ $12.2 \lambda)$

\begin{tabular}{ccccccccccc}
\hline Decentering Ratio $L$ & 0.10 & 0.20 & 0.30 & 0.40 & 0.50 & 0.60 & 0.70 & 0.80 & 0.90 & 1.00
\end{tabular}
\begin{tabular}{lllllllllll} 
Slippage Amount $\Delta r(\lambda)$ & 0.05 & 0.11 & 0.15 & 0.21 & 0.27 & 0.32 & 0.37 & 0.42 & 0.49 & 0.53 \\
\hline
\end{tabular}

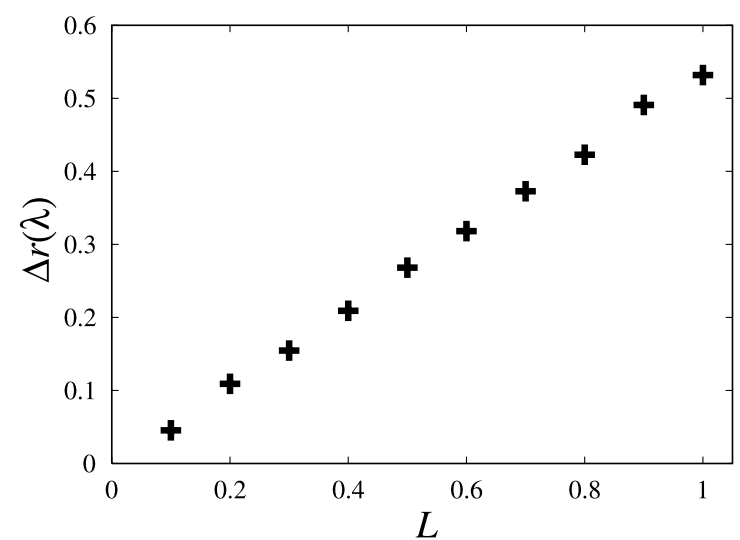

Fig. 7 Plot of $L$ and slippage amount from $r=0$ of each radiated beam, $\Delta r$ (The average of eleven calculation results around $z=12.2 \lambda$ )

it is shown that the propagation paths of radiated beams are shifted in the vicinity of the sound source, and that the radiating positions of the beam are scanned.

Table 2 shows the slippage amount from $r=0$ of the radiated beam, $\Delta r$, for each array in which $L$ is made to change from 0 to 1 in 0.1 intervals, and Fig. 7 shows the plot of the relationship between $L$ and $\Delta r$ shown in Table 2. The results used in Table 2 and Fig. 7 are the ones calculated using intervals of $0.05 \lambda$, in the $r$-axial direction, and $0.1 \lambda$, in the $z$-axial direction. In Table 2 and Fig. $7, z$ is set to $12.2 \lambda$, the vicinity of the farthest extreme value of radiated beams, and the results are smoothed by taking the average with neighboring five calculation results on both sides of the result at $z=12.2 \lambda$.

From Table 2 and Fig. 7, the following appear. When the calculation interval in the $r$-axial direction is considered, $\Delta r$ in the case of the decentering ratio $L=1.0$ almost agrees with 0.52765881 , the maximum possible value of the decentering amount in the second array element, $a_{2}-b_{1}$. In the other case, $\Delta r$ is almost proportional to $L$ within 0 and the value in the case of $L=1.0$. As the result, using the size of the clearance between decentering array element as the proportion coefficient, $\Delta r$ is expressed by the following equation, as well as the actual decentering amount:

$$
\Delta r=\left(a_{2}-b_{1}\right) \cdot L .
$$

These results shown in Table 2 and Fig. 7 are different from the results obtained in the previous calculations ${ }^{(11)-(17)}$. The beam is not steered but shifted, and the range of shifting becomes smaller than the wavelength. However, the method of decentering described in this paper is different from that of the past. In the conventional methods, decentering all, or multiple ring elements in the array were made to be an assumption. In the presented method, on the other hand, only one ring element that is arranged next to the central disk element is decentered. Therefore, though the slippage is generated at the vicinity of the sound source, it is not generated at other part of the beam formed by the not-decentered array element.

This method is an effective one in the point that the procedure of the decentering is greatly simplified, even if the range of shifting is small.

\section{Conclusions}

By using an annular transducer array that introduces a decentering operation, a method for realizing a sound source with a scanning function for the radiated acoustic beam is presented. In the presented method, the decentering operation is optimally. Calculations of the radiated sound pressure magnitude distributions with annular transducer arrays of which only one ring element neighboring upon the center disk element is decentered are carried out. From the results of the calculation, the following are shown. The beams radiated from the decentered arrays have sharp profiles and amplitude distributions that fairly agree with the case of the nondecentered array, the propagation paths of the beams are shifted in the vicinity of the sound source, and the slippage amount of the center of each beam is proportional to the decentering ratio of the decentered element. By synthesizing these results, it is confirmed that the scanning function of the radiating position of the beam is incorporated into the annular array sound source by the concise decentering operation.

The technique presented in this paper is expected to be applicable to sound sources for directional loudspeakers, probes for precise acoustic imaging, holding or controlling microobjects, and so forth. In the beam used in these applications, it is considered that precisely beam shifting is more important than increasing the amount of the beam shifting. Especially, there seems to be the tendency in dynamic applications. In the calculation result shown in this paper, it is considered that the condition for using to the applications described in advance is satisfied, though the maximum amount of the beam shifting is a half-wavelength. Furthermore, this method is the most 
simplified one, in comparison with the conventional decentering method for an annular transducer array, so its applicability seems to be high in the production of a practical sound source with a scanning function.

We recognize that bringing out the behavior of the beam in the mathematical manner completely is the problem that should continuously carry out the examination and solve, including the case in which multiple elements are decentered. Accordingly, we will conduct further examinations, including the examination of the method for realizing the sound source using this technique.

\section{Acknowledgement}

This work was supported in part by a Grant-in-Aid for Scientific Research C (No. 15560355) from the Japan Society for the Promotion of Science.

\section{References}

(1) Durnin, J., Exact Solutions for Nondiffracting Beams. I. The Scalar Theory, J. Opt. Soc. Am. A, Vol.4, No.4 (1987), pp.651-654.

( 2 ) Durnin, J., Miceli, J.J., Jr. and Eberly, J.H., DiffractionFree Beams, Phys. Rev. Lett., Vol.58, No.15 (1987), pp.1499-1501.

( 3 ) Hsu, D.K., Margetan, F.J. and Thompson, D.O., Bessel Beam Ultrasonic Transducer: Fabrication Method and Experimental Results, Appl. Phys. Lett., Vol.55, No.20 (1989), pp.2066-2068.

( 4 ) Lu, J.-Y. and Greenleaf, J.F., Ultrasonic Nondiffracting Transducer for Medical Imaging, IEEE Trans. Ultrason., Ferroelectr. \& Freq. Control, Vol.37, No.5 (1990), pp.438-447.

( 5 ) Campbell, J.A. and Soloway, S., Generation of a Nondiffracting Beam with Frequency-Independent Beamwidth, J. Acoust. Soc. Am., Vol.88, No.5 (1990), pp.2467-2477.

( 6 ) Nagai, K., Monma, H. and Mizutani, K., Calculation of Acoustic Near Field from Bessel Beam Transducers with Annular Transducer Array, Jpn. J. Appl. Phys., Vol.32, No.5B (1993), pp.2295-2297.

( 7 ) Koike, T., Yamada, K. and Nakamura, K., Characteristics of a Discretely Weighted Conical Transducer for Generation of Limited Diffraction Beams, Jpn. J. Appl.
Phys., Vol.35, No.5B (1996), pp.3184-3186.

( 8 ) Holm, S., Bessel and Conical Beams and Approximation with Annular Arrays, IEEE Trans. Ultrason., Ferroelectr. \& Freq. Control, Vol.45, No.3 (1998), pp.712718.

( 9 ) Yokoyama, T., Masuyama, H., Nagai, K., Mizutani, K. and Hasegawa, A., Nondiffraction Beam Generated from an Annular Array Driven by Uniform Velocity Amplitude, Proc. 1998 IEEE Int. Ultrason. Symp., (1998), pp.1069-1072.

(10) Masuyama, H., Yokoyama, T., Nagai, K. and Mizutani, K., Generation of Bessel Beam from EquiamplitudeDriven Annular Transducer Array Consisting of a Few Elements, Jpn. J. Appl. Phys., Vol.38, No.5B (1999), pp.3080-3084.

(11) Masuyama, H., Mizutani, K. and Nagai, K., Sound Source with Direction-Variable Beam Using Annular Transducer Array, Proc. 2002 IEEE Int. Ultrason. Symp., (2002), pp.1069-1072.

(12) Masuyama, H., Mizutani, K. and Nagai, K., DirectionVariable Type Sound Source by Annular Transducer Array, Proc. VS Tech 2003, (in Japanese), (2003), pp.140-143.

(13) Masuyama, H., Mizutani, K. and Nagai, K., Decentered Annular Transducer Array for Generating Direction-Variable Acoustic Beam, Proc. 16th World Conf. Nondest. Test., CD-ROM, (2004).

(14) Masuyama, H., Mizutani, K. and Nagai, K., Correspondence between Individual Decentered Element and Alteration of Beam Direction in Annular Array Sound Source, Proc. 18th Int. Cong. Acoust., Vol.1 (2004), pp.671-674.

(15) Masuyama, H., Mizutani, K. and Nagai, K., Effect of Decentering Ratio Fluctuation of Annular Array Element on Direction-Variable Beam, Proc. Symp. Ultrason. Electron., (in Japanese), Vol.24 (2003), pp.17-18.

(16) Masuyama, H., Mizutani, K. and Nagai, K., Effect of Decentering Ratio Fluctuation of Annular Array Element in Direction-Variable Type Sound Sources, Jpn. J. Appl. Phys., Vol.43, No.5B (2004), pp.2822-2826.

(17) Masuyama, H., Mizutani, K. and Nagai, K., Effect of Decentering Ratio of Elements in a Variable Radiation Direction Sound Source on Beam Profile, Acoust. Sci. \& Tech., Vol.25, No.5 (2004), pp.386-388. 